\title{
Ape1 regulates hematopoietic differentiation of embryonic stem cells through its redox functional domain
}

\author{
Gang-Ming Zou ${ }^{1,2}$, Mei-Hua Luo ${ }^{1,2}$, April Reed ${ }^{1}$, Mark R. Kelley ${ }^{1,2,3,4}$, \\ Mervin C. Yoder ${ }^{1,2,4}$ \\ Department of Pediatrics (Section of Hematology/Oncology) ${ }^{1}$, Herman B Wells Center for \\ Pediatric Research ${ }^{2}$, Department of Pharmacology and Toxicology ${ }^{3}$, and Department of \\ Biochemistry \& Molecular Biology ${ }^{4}$, Indiana University School of Medicine, 1044 W. Walnut, \\ Indianapolis, IN 46202
}

Short title for running heading: ES cell-derived hematopoiesis is regulated by Ape1

Author contributions:

Gang-Ming Zou designed and performed research, analyzed data, and wrote paper

Mei-Hua Luo performed research

April Reed performed research

Mark R. Kelley designed research, analyzed data, wrote paper

Mervin C. Yoder designed research, analyzed data, and wrote paper

Research Grant / Financial Support: This research was supported in part by HL63069 (MY) and CA094025, CA106298 ES03456 and P30 CA82709 (MK).

Word counts: $\quad$ Abstract: $194 \quad$ Words: 2,842

Scientific Heading: Hematopoiesis

Corresponding Author:

Mervin C Yoder, MD

Herman B Well Center for Pediatrics Research

Indiana University School of Medicine

1044 West Walnut St

Indianapolis IN 46202

Tel: $317-274-4738$

Fax: 317-274 8928

Email: myoder@iupui.edu 


\begin{abstract}
Ape1 is a molecule with dual functions in DNA repair and redox regulation of transcription factors. In Ape1 deficient mice, embryos do not survive beyond embryonic day 9, indicating that this molecule is required for normal embryo development. Currently, direct evidence of the role of Ape1 in regulating hematopoiesis is lacking. We used the ES cell differentiation system and a siRNA approach to knock down Ape1 gene expression to test the role of Ape1 in hematopoiesis. Hemangioblast development from ES cells was reduced 2 to 3 fold when Ape1 gene expression was knocked down by Ape1 specific siRNA, as was primitive and definitive hematopoiesis. Impaired hematopoiesis was not associated with increased apoptosis in siRNA treated cells. To begin to explore the mechanism whereby Ape1 regulates hematopoiesis, we found that inhibition of the redox activity of Ape1 with E3330, a specific Ape1 redox inhibitor, but not Ape1 DNA repair activity which was blocked using the small molecule, methoxyamine, affected cytokine-mediated hemangioblast development in vitro. In summary, these data indicate Ape1 is required in normal embryonic hematopoiesis and that the redox, but not the repair endonuclease function of Ape1 is critical in normal embryonic hematopoietic development.
\end{abstract}




\section{Introduction}

Ape1/Ref-1 is a multifunctional protein involved in apurinic/apyrimidinic endonuclease DNA base excision repair activity, in proofreading exonuclease activity, and in modulating DNA binding activity of several transcription factors including NFkB, Egr-1, p53, AP-1, CREB, HIF- $\alpha$ and members of the Pax family ${ }^{1}$. Ape1 acts on apurinic/apyrimidinic (AP) sites in DNA as a major member of the base excision repair (BER) pathway and is involved in oxidative DNA damage repair ${ }^{2}$. Ape1 is ubiquitously expressed at high basal levels and in previous studies to determine the function of Ape1 in vivo, Ape1 was disrupted using a gene targeting strategy ${ }^{3}$. Embryos lacking expression of Ape1 die in utero between implantation and day 6.5, indicating that Ape1 is required for normal embryonic development. However, this approach does not allow for the determination of whether it is the redox or repair function of Ape1 that is crucial for embryonic development. Furthermore, this early lethality precludes analysis of the role of Ape1 in hematopoiesis.

Mouse embryonic stem (ES) cells are derived from the inner cell mass of the 3.5-dayold blastocyst ${ }^{4,5}$. ES cells retain the differentiation ability of the inner cell mass after many passages in the presence of leukemia inhibitory factor (LIF) ${ }^{6}$. Removal of LIF results in ES cell differentiation into embryoid bodies $(\mathrm{EB})^{7}$. Using the ES/EB system, a precursor that responds to vascular endothelial growth factor (VEGF) and generates colonies consisting of undifferentiated blast cells was identified ${ }^{8}$. These VEGFresponsive blast cell colonies were shown to contain endothelial, primitive erythroid, and various definitive hematopoietic precursors. These cells are referred to as blast colony-forming cells (BL-CFC) and are thought to be cells that represent the hemangioblast precursor of blood and endothelial lineages. 
Ape1 siRNA has previously been used to knock down target gene expression in several cell types ${ }^{9-11}$. In this study, we used siRNA to knock down Ape1 gene expression in EB cells and discovered that reduction of Ape1 by siRNA results in a significant decrease in the frequency of hemangioblast formation and diminished formation of primitive and definitive hematopoietic colonies. Additionally, using an Ape1 specific redox inhibitor, E3330, we demonstrate that it is the redox function of Ape1 and not the repair activity that is involved in hematopoietic differentiation. These data suggest that Ape1 may play a critical role in embryonic hematopoiesis 


\section{Materials and method}

\section{Cells and reagents}

The mouse CCE ES cells (passage No.10) were kindly provided by Bill Carter in the mouse Transgene and knockout core facility in our institute. Cytokines LIF, VEGF, SCF, FGF and methylcellulose-based ES cell differentiation medium and collagenase were purchased from Stem Cell Inc. (Vancouver, BC, Canada). Ape1 antibodies were purchased from Novus Biological (Littleton, CO, USA). FITC labeled anti-mouse Flk-1 mAb was purchased from BD Pharmingen Inc (San Diego, CA, USA). Oligofectamine 2000 was purchased from Invitrogen (Carlsbad, CA, USA). All Ape1 dsRNA and scrambled Ape1 siRNAs were commercially obtained from Dharmacon (Lafayette, CO, USA). The 21-base sequence was subjected to a BLAST-search (NCBI) database of EST libraries to ensure that only one gene was targeted. Sense strand of RNA oligonucleotides correspond to Ape1 (GTCTGGTAAGACTGGAATACC) was chemically synthesized using standard methods and HPLC purification (Dharmacon Inc. Lafayette, CO USA). E3330, the redox specific inhibitor of Ape1 molecule, was

provided by Dr. Rick Borch and Rod Nyland in Department of Medical Chemistry, Purdue University (Lafayette, IN, USA)

\section{In vitro differentiation of ES cells}

CCE ES cells were maintained on gelatinized tissue culture dishes $(100 \mathrm{~mm}$; Costar, Cambridge, MA, USA) in standard ES culture medium consisting of Dulbecco's modified Eagle's medium (DMEM) supplemented with $15 \%$ fetal calf serum (FCS; GIBCO, Grand Island, NY, USA), $0.1 \mathrm{mM}$ L-glutamine, $150 \mathrm{mM}$ monothioglycerol (MTG), penicillin $100 \mathrm{U} / \mathrm{mL}$, streptomycin $100 \mathrm{mg} / \mathrm{mL}$, and LIF $1000 \mathrm{U} / \mathrm{mL}$. The culture medium was changed every day, and cells were passaged every 2 or 3 days ${ }^{12}$. 
To initiate differentiation into embryonic bodies (EBs), dissociated ES cells were added to IMDM medium (Invitrogen co,), $15 \%$ FBS (Fetal bovine serum) (HCC6900, Stem Cell Technologies, Vancouver, BC, Canada), 100 ng/ml stem cell factor (R\&D System, Minneapolis, MN, USA) and $450 \mu \mathrm{M}$ monothioglycerol (Sigma, St. Louis, MO, USA) at a cell concentration of $5 \sim 10 \times 10^{3}$ cells / $\mathrm{ml}$ plated in a $100 \mathrm{~mm}$ low adhesion dishes.

\section{Harvesting EBs}

EBs were removed from liquid medium IMDM. 3 day aged EBs were collected, washed twice with PBSA (PBS with $1 \%$ albumin), and resuspended in $0.25 \%$ (wt/vol) Trypsin mix in $15 \%$ FBS containing PBSA. The EBs were incubated at $37{ }^{\circ} \mathrm{C}$ for 5 minutes and dissociated into a single-cell suspension by passing through a 20-gauge needle. Remaining small aggregates were removed by filtration through a $40-\mu \mathrm{m}$ mesh (Falcon). EB cells were spun and resuspended in $0.5 \%$ BSA (Path-O-Cyte 4; Miles, Kankakee, IL, USA) in PBSA at concentration of $5 \times 10^{6}$ cells $/ \mathrm{ml}$.

\section{Transfection of EB cells with oligos of dsRNA}

Transient transfection of siRNAs was carried out as we previously reported ${ }^{7,13}$. Total EB cells were diluted with fresh medium without antibiotics and transferred to 12-well

plates at $1 \times 10^{5}$ cells/well $(500 \mu 1$ per well). Transient transfection of dsRNAs was carried out using Oligofectamine 2000 (Life Technologies, Carlsbad, CA, USA). Cells were untreated (control), or treated with scrambled Ape1 siRNA (25 50nM), or Ape1 siRNA (25 50nM). 


\section{Clonogenic cell assay}

For hemangioblast assay, after siRNA transfection, day-3 EB cells were mixed with IMDM, 20\% FCS, 10\% BSA (Stem Cell Technology), $100 \mu \mathrm{g} / \mathrm{ml}$ bovine transferrin, 10ng/ml bovine insulin, $0.1 \mathrm{mmol} / \mathrm{L}$ MTG, $1 \%$ methylcellulose, and distributed to $35-$ mm bacterial-grade dishes at $10^{4}$ cells/plate with VEGF, SCF, DT4 conditional medium (CM). For primitive erythroid cell (Ery-P) assay, day 6 EB cells were cultivated with EPO at $5 \mathrm{IU} / \mathrm{ml}$ as previously described ${ }^{14}$. For granulocyte-macrophage (CFU-GM) colony assay, day 10 EB cells were cultured with granulocyte-macrophage colonystimulating factor (GM-CSF) at $10 \mathrm{ng} / \mathrm{ml}$ and macrophage colony-stimulating factor (M-CSF) at $5 \mathrm{ng} / \mathrm{ml}^{14}$.

\section{$R T-P C R$}

Total RNA was extracted from undifferentiated ES cells and EB cell populations by using TRIZOL (Invitrogen. USA), Reverse transcription was performed using Superscript II reverse transcriptase (GIBCO BRL, Carlsbad, CA, USA), and PCR was performed using Pyrobest DNA polymerase. Primer sequences for Brachyury, Flk-1, and $s c l$ genes have been reported ${ }^{8}$. The primer sequence of $\beta$-actin gene has been reported $^{15}$.

\section{Western blot analysis}

Whole cell extracts were prepared using nuclear protein extract buffer (Laribee and Klemsz, 2001) from both Ape1-siRNA or scrambled siRNA treated and untreated cells. The cell lysates were separated by SDS-polyacrylamide electrophoresis using a $10 \%$ (w/v) polyacrylamide resolving gel and transferred electrophoretically to a nitrocellulose membrane. The blots were blocked with 5\% TBS/T buffer for $1 \mathrm{hr}$. 
Immunobloting was performed using the Ape1 primary monoclonal antibody (Novus Biologicals, Littleton, CO, USA) at a 1:400 dilution at room temperature, and the peroxidase-conjugated secondary Abs (Amersham Pharmacia, USA) over night at $4{ }^{\circ} \mathrm{C}$. This antibody has been extensively used by us and others ${ }^{11,16-20}$ All immunoblots were visualized by enhanced chemiluminescence (ECL) (Amersham Pharmacia Biotech, USA).

Inhibition of DNA repair domain of Ape1 with MX and redox domain with E3330. E3330 is a specific inhibitor of redox activity of Ape1 protein, while MX is a specific inhibitor of the DNA repair (AP endonuclease) function of Ape1 protein. MX does not specifically bind to Ape1, but binds to the abasic site that is recognized by Ape1 and prevents Ape1's ability to cleave the AP site ${ }^{21-25}$.

E3330 has been shown to be a very specific inhibitor of Ape1 redox function. Farwestern blots, and binding assays between radiolabeled E3330 and proteins renatured on membrane blots, demonstrated that ${ }^{14} \mathrm{C}$-labeled E3330 specifically bound to both recombinant APE1 and purified APE1 from cell nuclear extracts ${ }^{26}$. The binding constant of APE1 and E3330 was estimated by surface plasmon resonance analysis (SPR) resulting in a kinetic constant value of $1.6 \mathrm{nM}$ which suggests a specific interaction between APE1 and E3330 ${ }^{26}$. Therefore, E3330 is a specific inhibitor of APE1 redox function. E3330 does not affect the repair function of Ape1 (Kelley and Luo, unpublished data).

To verify the role of Ape1 in hemangioblast development via redox domain or DNA repair domain, various doses of E3330 (10 -100 $\mu \mathrm{M})$ or MX (1-10mM) were added to 
standard hemangioblast culture. Hemangioblast colonies were scored after 4 days of culture.

\section{Cell cycle analysis}

EB cells were treated with either scrambled siRNA or Ape1 siRNA. After 48 hours of culture, cells were washed with PBS, and resuspended in $400 \mu \mathrm{L}$ of hypotonic buffer $(0.15 \%$ Triton-X100 and $20 \mu \mathrm{g} / \mathrm{ml}$ of RNase A) containing $50 \mu \mathrm{g} / \mathrm{ml}$ of Propidium Iodide ( PI), and analyzed using FACScan (BD Bioscience, Mountain view, CA). 


\section{Results}

Ape1 is expressed in both undifferentiated and differentiated ES cells

To examine whether Ape1 is expressed in ES cells and differentiated ES cells, we isolated total cell protein from ES cells, and day 3, 6, or 10 EB cells. Western blot analysis showed that Ape1 protein is detectable in both ES cells and EB cells (Fig.1A). Further experiments showed that Ape1 siRNA, but not scrambled control siRNA, is effective to knockdown Ape1 gene expression in EB cells when probed by western blot analysis after $48 \mathrm{~h}$ of siRNA transfection (Fig.1B).

\section{Normal hemangioblast development from ES cell development}

ES cells differentiate to EBs in the absence of LIF in culture. Day 3 EB cells replated into culture wells with VEGF and SCF results in the emergence of hemangioblast colonies. Some residual undifferentiated ES cells in EB were able to differentiate into a cell mass called secondary EBs, that are round bodies with a smooth edge and are easy to distinguish from hemangioblast colonies (Fig. 2A). We separately collected BL-

CFCs or $2^{\text {nd }}$ EBs and RT-PCR analysis revealed that they differ in their gene expression pattern (Fig. 2B). Knockdown of Ape1 gene expression results in a decrease of BLCFC formation and an increase in the $2^{\text {nd }} E B$ numbers in the culture (Fig. 2C,D).

\section{Apel is essential for both primitive and definitive hematopoiesis}

To test the role of Ape1 in primitive erythroid progenitor (Ery-P) cell development, day 6 EBs were dissociated and EB cells were replated in methylcellulose culture medium supplemented with plasma derived serum and with erythropoietin. Knockdown of Ape1 expression in day $6 \mathrm{~EB}$ cells blunted their potential to form Ery-P (Figure 3A). To determine whether a reduction of Ape1 expression affected definitive hematopoiesis, 
Ape1 siRNA was introduced into dissociated day 10 EB cells followed by progenitor assays. Cells with reduced Ape1 expression generated significantly fewer definitive erythroid colonies (Ery-D) compared to untreated cells or cells transfected with scrambled siRNA (Figure 3B). Moreover, to examine myeloid differentiation, CFUGMs were scored by replating control or Ape1 siRNA-treated day 10 EB cells in the presence of Epo, SCF, GM-CSF and IL-3. Our results indicated that the frequencies of CFU-GM colony were decreased in Ape1 siRNA-transfected group compared with the control scrambled siRNA-transfected group (Fig.3C) $(\mathrm{P}<0.05)$. Consistent with the levels of Ape1 expression detected by Western blot analysis in cells transfected with 25 $\mathrm{nM}$ and $50 \mathrm{nM}$ Ape1 siRNA (Figure 1B), no significant difference in hematopoietic colony frequency between cells treated with $25 \mathrm{nM}$ or $50 \mathrm{nM}$ of Ape1 siRNA was observed (Figure 3A, 3B, and 3C). As $\mathrm{CD}^{+} 4^{+}$day $10 \mathrm{~EB}$ cells contain hematopoietic progenitor cells $^{7,13}$, we then sorted CD34 ${ }^{+}$EB cells from day 10 EBs and treated cells with scrambled or Ape1 siRNA. Our data revealed that knockdown of Ape1 gene expression in these cells reduced their ability to form colonies of Ery-D, CFU-GM and CFU-Mix (Fig. 4A, B, C).

Knockdown of Ape1 gene expression in EB cells did not induce apoptosis in these cells To determine whether or not knockdown of Ape1 gene expression in EB cells induced apoptosis, siRNA transfected cells were harvested after 48 hours of transfection and stained with Annexin V and PI. The cells were then analyzed by FACS. As shown in Figure 5, knockdown of Ape1 gene expression with siRNA did not trigger apoptosis in EB cells. In contrast, cells treated with IFN- $\gamma$ displayed robust apoptosis (Fig. 5).

Inhibition of Ape1 redox activity by E3330 but not DNA repair activity by 
methoxyamine affects hemangioblast development in vitro

To address which functional domain in the Ape1 molecule may play the major role in regulation of hematopoiesis, we added the Ape1 redox inhibitor E3330 (0 - $100 \mu \mathrm{M})$ or the Ape1 abasic site excision inhibitor, $\mathrm{MX}(0 \sim 10 \mathrm{mM})$ to add to the cultured EB cells. Inhibition of Ape1 redox activity by E3330, but not Ape1 DNA repair activity by MX affected cytokine-mediated hemangioblast development in vitro (Fig.6). Furthermore, the inhibition of BL-CFC and Ery-P colony formation via E3330 was dose dependent.

Knock down of Ape1 in EB cells induces G1 arrest in EB cells

To examine whether or not the decrease in frequency of hematopoietic colonies was caused by low cell proliferation, we performed cell cycle analysis of the cells treated with Ape1 siRNA. As shown in Figure 7, reduction of Ape1 gene expression with Ape1 siRNA, but not scrambled siRNA, significantly increased day 3 EB cells or Flk- $1^{+}$EB cells in the G1 phase and decreased the percent of cells in S phase. This indicates that knockdown of Ape1 gene expression in EB cells resulted in a decrease in the G1/S transition of the cell cycle in EB cells. 


\section{Discussion}

We have demonstrated that the knockdown of Ape1 gene expression in mouse ES cells results in a decrease in the formation of hemangioblast, primitive, and definitive hematopoietic colony frequencies. Our study also reveals that knockdown of Ape1 gene expression in EB cells affected the G1/S transition of the cell cycle in these cells. Further studies showed that only inhibition of Ape1 redox activity, but not Ape1 DNA repair activity diminished hematopoiesis.

ES cell differentiation is a valuable system to study molecular regulation in embryonic hematopoietic development. In the absence of LIF, ES cells differentiate into EBs after a 3 day differentiation ${ }^{27}$. Day 3 EB cells include Flk- $1^{+}$BL-CFC cells that form colonies in culture when VEGF is supplied ${ }^{8}$. Flk1 and SCL are molecular determinants of the hemangioblast and expression of both these molecules is required for colony formation $^{28}$. BL-CFC represent the in vitro equivalent of the hemangioblast and, as such, one of the earliest stages of hematopoietic development described to date. Functional studies have demonstrated that some of these blast colonies contain primitive and definitive hematopoietic precursors as well as precursors that give rise to adherent endothelial-like cells. Hemangioblast development can be regulated by a number of factors. TPO alone supported BL-CFC formation and nearly doubled the number of BL-CFC when added together with VEGF and $\mathrm{SCF}^{29}$. The homeoprotein Hex is essential for hemangioblast differentiation into definitive embryonic hematopoietic progenitors ${ }^{30}$. We recently described the positive regulatory role of the protein tyrosine phosphatase Shp-2 in hemangioblast development and implicated Shp2 as an important mediator of FGF augmentation of BL-CFC activity in vitro ${ }^{14}$. In the present study, we identified a novel role of Ape1 as a regulatory molecule in 
hemangioblast development. The reduction in BL-CFC resulting from decreased Ape1 expression in the EB, may result in diminished production of both primitive and definitive hematopoietic progenitors. It is interesting to contemplate that knockdown of Ape1 expression with siRNA may have affected emergence of the BL-CFC from mesoderm whereas, use of the Ape1 redox inhibitor molecule E3330 may have had a more direct effect on differentiation of the BL-CFC toward hematopoietic progenitors. To address whether a reduction in Ape1 expression directly influences progenitor differentiation we isolated and knocked down Ape1 expression in this cell population that contains primary hematopoietic progenitor cells.

Changes in expression of Ape1 have been correlated with myeloid differentiation in HL60 leukemic cells induced by RA or $\mathrm{DMSO}^{31}$. However, the regulatory role of Ape1 in myeloid differentiation of primary hematopoietic stem/progenitor cells has not examined. Our study clearly demonstrated that Ape1 is essential for GM-CSF and other cytokines to stimulate myeloid hematopoiesis, as knockdown of Ape1 expression in CD34+ cells resulted in significant reduction of erythroid and myeloid progenitors in vitro. Therefore, we propose that Ape1 regulates normal erythromyelopoiesis.

In previous studies, PDGF-BB stimulated cell cycle progression from G0/G1 to $\mathrm{S}$ in smooth muscle cells, with one of the postulated steps being redox-regulation of AP-1 by Ape1 protein $^{32}$. Therefore, we wanted to see if Ape1 may play a role in growth factor stimulated cell cycle regulation. We report here that Ape1 regulates cell cycle status in hematopoietic progenitors and that Ape1 also positively regulate G1/S transition in EB cells. The cell cycle arrest of Ape1 siRNA treated EB cells may be one mechanism to explain diminished hematopoietic CFC activity reported here. Future studies will need 
to prove how Ape1 may regulate cyclin dependent kinases and cyclins that are critical for G/S transition.

In conclusion, this study presents direct evidence that Ape1 positively regulates embryonic hematopoiesis through its redox function. However, the target of Ape1's redox signaling has not been identified by this type of analysis and additional studies will need to address which downstream target or targets are involved in this pathway. As this study was limited to investigation of the regulatory role of Ape1 in embryonic hematopoiesis using the ES cell system, further studies to identify a possible role for Ape1 in adult myelolymphoid development may prove insightful. 


\section{Acknowledgement:}

We thank Bill Carter in transgene center to offer us CCE ES cells. We would like to thank Rod Nyland II and Rick Borch in Department of Medical Chemistry, Purdue University Cancer Center for offering us the E3330. 


\section{References:}

1. Merluzzi S, Moretti M, Altamura S, et al. CD40 stimulation induces Pax5/BSAP and EBF activation through a APE/Ref-1-dependent redox mechanism. J Biol Chem. 2004;279:1777-1786.

2. Evans AR, Limp-Foster M, Kelley MR. Going APE over ref-1. Mutat Res. 2000;461:83-108.

3. Xanthoudakis S, Curran T. Redox regulation of AP-1: a link between transcription factor signaling and DNA repair. Adv Exp Med Biol. 1996;387:69-75.

4. Evans MJ, Kaufman MH. Establishment in culture of pluripotential cells from mouse embryos. Nature. 1981;292:154-156.

5. Martin GR. Isolation of a pluripotent cell line from early mouse embryos cultured in medium conditioned by teratocarcinoma stem cells. Proc Natl Acad Sci U S A. 1981;78:7634-7638.

6. Sato M, Nakano T. Embryonic stem cell. Intern Med. 2001;40:195-200.

7. Zou GM, Wu W, Chen J, Rowley JD. Duplexes of 21-nucleotide RNAs mediate RNA interference in differentiated mouse ES cells. Biol Cell. 2003;95:365-371.

8. Kennedy M, Firpo M, Choi K, et al. A common precursor for primitive erythropoiesis and definitive haematopoiesis. Nature. 1997;386:488-493.

9. Fan Z, Beresford PJ, Zhang D, et al. Cleaving the oxidative repair protein Ape1 enhances cell death mediated by granzyme A. Nat Immunol. 2003;4:145-153.

10. Fung H, Demple B. A vital role for Ape1/Ref1 protein in repairing spontaneous DNA damage in human cells. Mol Cell. 2005;17:463-470.

11. Wang D, Luo M, Kelley MR. Human apurinic endonuclease 1 (APE1) expression and prognostic significance in osteosarcoma: enhanced sensitivity of 
osteosarcoma to DNA damaging agents using silencing RNA APE1 expression inhibition. Mol Cancer Ther. 2004;3:679-686.

12. Zou GM, Chen JJ, Ni J. LIGHT induces differentiation of mouse embryonic stem cells associated with activation of ERK5. Oncogene. 2006;25:463-469.

13. Zou GM, Chen JJ, Yoder MC, Wu W, Rowley JD. Knockdown of Pu.1 by small interfering RNA in CD34+ embryoid body cells derived from mouse ES cells turns cell fate determination to pro-B cells. Proc Natl Acad Sci U S A. 2005;102:13236-13241.

14. Zou GM, Chan RJ, Shelley WC, Yoder MC. Knockdown of Shp-2 by siRNA on mouse embryonic stem cell derived EB cells affects hematopoietic development. Stem Cells. 2006; 24:587-594.

15. Olson MC, Scott EW, Hack AA, et al. PU. 1 is not essential for early myeloid gene expression but is required for terminal myeloid differentiation. Immunity. 1995;3:703-714.

16. Vasko MR, Guo C, Kelley MR. The multifunctional DNA repair/redox enzyme Ape1/Ref-1 promotes survival of neurons after oxidative stress. DNA Repair (Amst). 2005;4:367-379.

17. Tell G, Damante G, Caldwell D, Kelley MR. The intracellular localization of APE1/Ref-1: more than a passive phenomenon? Antioxid Redox Signal. $2005 ; 7: 367-384$.

18. Pines A, Perrone L, Bivi N, et al. Activation of APE1/Ref-1 is dependent on reactive oxygen species generated after purinergic receptor stimulation by ATP. Nucleic Acids Res. 2005;33:4379-4394.

19. Freitas S, Moore DH, Michael H, Kelley MR. Studies of apurinic/apyrimidinic endonuclease/ref-1 expression in epithelial ovarian cancer: correlations with 
tumor progression and platinum resistance. Clin Cancer Res. 2003;9:46894694.

20. Tell G, Pines A, Paron I, et al. Redox effector factor-1 regulates the activity of thyroid transcription factor 1 by controlling the redox state of the $\mathrm{N}$ transcriptional activation domain. J Biol Chem. 2002;277:14564-14574.

21. Rinne M, Caldwell D, Kelley MR. Transient adenoviral N-methylpurine DNA glycosylase overexpression imparts chemotherapeutic sensitivity to human breast cancer cells. Mol Cancer Ther. 2004;3:955-967.

22. Liu L, Gerson SL. Therapeutic impact of methoxyamine: blocking repair of abasic sites in the base excision repair pathway. Curr Opin Investig Drugs. 2004;5:623-627.

23. Taverna P, Hwang HS, Schupp JE, et al. Inhibition of base excision repair potentiates iododeoxyuridine-induced cytotoxicity and radiosensitization. Cancer Res. 2003;63:838-846.

24. Liu L, Nakatsuru Y, Gerson SL. Base excision repair as a therapeutic target in colon cancer. Clin Cancer Res. 2002;8:2985-2991.

25. Taverna P, Liu L, Hwang HS, Hanson AJ, Kinsella TJ, Gerson SL. Methoxyamine potentiates DNA single strand breaks and double strand breaks induced by temozolomide in colon cancer cells. Mutat Res. 2001;485:269-281.

26. Shimizu N, Sugimoto K, Tang J, et al. High-performance affinity beads for identifying drug receptors. Nat Biotechnol. 2000;18:877-881.

27. Chan RJ, Johnson SA, Li Y, Yoder MC, Feng GS. A definitive role of Shp-2 tyrosine phosphatase in mediating embryonic stem cell differentiation and hematopoiesis. Blood. 2003;102:2074-2080. 
28. Chung YS, Zhang WJ, Arentson E, Kingsley PD, Palis J, Choi K. Lineage analysis of the hemangioblast as defined by FLK1 and SCL expression. Development. 2002;129:5511-5520.

29. Perlingeiro RC, Kyba M, Bodie S, Daley GQ. A role for thrombopoietin in hemangioblast development. Stem Cells. 2003;21:272-280.

30. Guo Y, Chan R, Ramsey H, et al. The homeoprotein Hex is required for hemangioblast differentiation. Blood. 2003;102:2428-2435.

31. Robertson KA, Hill DP, Xu Y, et al. Down-regulation of apurinic/apyrimidinic endonuclease expression is associated with the induction of apoptosis in differentiating myeloid leukemia cells. Cell Growth Differ. 1997;8:443-449.

32. He T, Weintraub NL, Goswami PC, et al. Redox factor-1 contributes to the regulation of progression from G0/G1 to S by PDGF in vascular smooth muscle cells. Am J Physiol Heart Circ Physiol. 2003;285:H804-812. 


\section{Figure Legends}

Fig.1. Ape1 siRNA is efficient to knockdown target gene expression in EB cells. A). Western blot analysis of Ape1 protein is expression both in ES and EB cells at different ages; days 3, 6 and 10. B). Silencing of endogenous Ape1 gene by Ape1 siRNA. Western blot analysis demonstrates that Ape1 siRNA efficiently knocks down Ape1 gene expression: lane 1: untreated cells; lane 2: cells treated with $25 \mathrm{nM}$ scrambled siRNA; lane 3: cells treated with Ape1 siRNA at $5 \mathrm{nM}$; lane 4: cells treated with Ape1 siRNA at $25 \mathrm{nM}$; lane 5. The cells were treated with Ape1 siRNA at $50 \mathrm{nM}$.

Fig.2. Knockdown of Ape1 expression results in a decreased frequency of BL-CFC formation. A) The distinct morphology between BL-CFC and $2^{\text {nd }}$ EB are depicted. (x20). B) The individual BL-CFC or $2^{\text {nd }}$ EB colonies were collected separately. mRNA was extracted and RT-PCR assay revealed the distinct phenotype between these two cell types; BL-CFC express Flk-1 and scl genes, but not the brachyury gene. In contrast, $2^{\text {nd }}$ EB cells express the brachyury gene, but not Flk-1 and scl genes. C) Hemangioblast progenitor assay indicated that knockdown of Ape1 gene expression with Ape1 siRNA, but not scrambled siRNA day 3 EB cells resulted in a decrease in BL-CFC formation. ${ }^{*} \mathrm{P}<0.05$ comparing Ape1 siRNA to scrambled siRNA, treatment with transfection reagent (OFA2000), or no treatment. Data represent the mean \pm SD for 3 experiments. D). Knockdown of Ape1 gene expression day 3 EB cells resulted in an increase in secondary EB formation in the culture. $* \mathrm{P}<0.05$ comparing Ape1 siRNA to scrambled siRNA, treatment with transfection reagent (OFA2000), or no treatment. Data represent the mean \pm SD for 3 experiments. 
Fig. 3. Reduction of Ape1 expression by siRNA abrogates primitive and definitive hematopoiesis. A) Cells derived from day $6 \mathrm{CCE}$ EBs were transfected with either Ape1 siRNA, or scrambled siRNA and plated for primitive progenitor assays (Ery-D). B) Cells derived from day $10 \mathrm{EBs}$ were transfected with $25 \mathrm{nM}$ or $50 \mathrm{nM}$ of Ape1 siRNA1, or scrambled siRNA and plated for either definitive erythroid progenitor assays (Ery-D) or C) granulocyte-macrophage colony assays (CFU-GM). At both 25 $\mathrm{nM}$ and $50 \mathrm{nM}$ of Ape1 siRNA in all assays conducted, significantly fewer progenitors developed. $* \mathrm{P}<0.05$ comparing cells transfected with Ape1 siRNA to that of scrambled siRNA for all progenitor assays conducted. Data represents the mean \pm SD for 3 experiments.

Fig. 4. Reduction of Ape1 expression by siRNA in CD34 $^{+}$EB10 cells abrogates definitive hematopoiesis. A) $\mathrm{CD}^{+} 4^{+}$cells derived from day $10 \mathrm{CCE}$ EBs were transfected with either Ape1 siRNA, or scrambled siRNA and plated for definitive erythroid progenitor ( Ery-D) assays. B) CD34 ${ }^{+} \mathrm{EB}$ cells derived from day 10 EBs were transfected with $25 \mathrm{nM}$ or $50 \mathrm{nM}$ of Ape1 siRNA, or scrambled siRNA and plated into granulocyte-macrophage colony (CFU-GM) assays, or $\mathbf{C}$ ) mixed lineage colony assays (CFU-Mix). At both $25 \mathrm{nM}$ and $50 \mathrm{nM}$ of Ape1 siRNA in all CD34 ${ }^{+} \mathrm{EB}$ cells derived from day10 EBs were transfected with $25 \mathrm{nM}$ or $50 \mathrm{nM}$ of Ape1 siRNA, or scrambled siRNA and plated into mixed progenitor colony (CFU-Mix) assay. There are significantly fewer progenitors developed. $* \mathrm{P}<0.05$ comparing cells transfected with Ape1 siRNA to that of scrambled siRNA for all progenitor assays conducted. Data represent the mean $\pm \mathrm{SD}$ for 3 experiments.

Fig. 5. Knockdown of Ape1 expression in EB3 cells or sorted CD34 ${ }^{+}$EB10 cells did not trigger apoptosis. $\mathrm{EB} 3$ or $\mathrm{CD}^{+} 4^{+}$day $10 \mathrm{~EB}$ cells were transfected with 
control siRNA or Ape1 siRNA, after 48 hours of transfection, cells were harvested and stained with Annexin V and PI. Double Annexin V and PI positive cells in staining are scored as apoptotic cell in FACS analysis. In a separate group, cells were treated with IFN- $\gamma(500 \mathrm{IU})$ as positive control in apoptosis induction. $(* \mathrm{P}<0.01)$

Fig. 6. Inhibition of redox activity of Ape1 but not base excision repair (BER) activity of Ape1 reduce BL-CFC and Ery-P formation. A). In the BL-CFC assay, EB 3 cells were treated with VEGF, SCF, and various dose of E3330 (0 to $100 \mathrm{nM}$ ) or MX (0 to $10 \mu \mathrm{M})$ as indicated in the culture. After 4 days culture, the BL-CFC colony are scored and the number is shown. Results shown are representative of three independent experiments, each performed in triplicate. Mean values significantly different from control cells (untreated or treated with DMSO) are indicated. $* \mathrm{P}<0.05$; $* * \mathrm{P}<0.01)$. B). In Ery-P assay, EB 6 cells were treated with Epo and various dose of E3330 (0 to $100 \mathrm{nM})$ or $\mathrm{MX}(0$ to $10 \mu \mathrm{M})$. After 7 days culture, Ery-P colony are scored. Results shown are representative of three independent experiments, each performed in triplicate. Mean values significantly different from control cell (untreated or treated with DMSO) are indicated. $* \mathrm{P}<0.05 ; * * \mathrm{P}<0.01)$.

Fig. 7. Ape1 regulates cell cycle G1-S transition in day 3 EB cells. Day 3 EB cells or Flk $-1^{+}$day 3 EB cells were treated with either transfection reagent (Oligofectamine 2000) alone, scrambled siRNA (25 nM) or Ape1 siRNA (25 nM) for 48 hours. Cells were collected, permeablized and stained with PI prior to flow cytometry to determine the percentage of cells in G1 and S phase. Open bars represent day 3 EB cells and solid black bars represent Flk-1 ${ }^{+}$day 3 EB cells. A). Percentage of G1 phase cells B). 
Percentage of $\mathrm{S}$ phase cells. Bars represent means $\pm \mathrm{SD}$ of triplicate experiments. $* \mathrm{P}<0.05$. 
Figure 1
A
ES d3EB d6EB d10EB

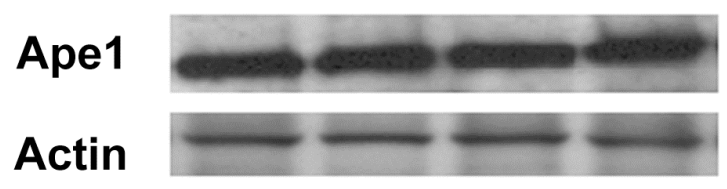
B

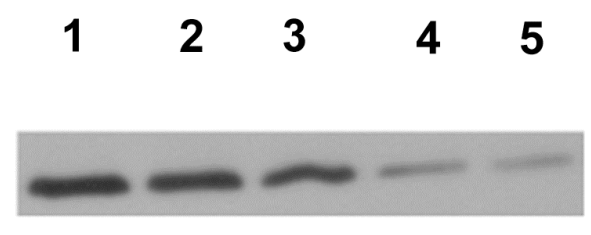
Ape1 siRNA

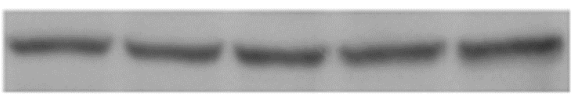
Scrambled siRNA
- $\quad-5 \mathrm{nM} 25 \mathrm{nM} 50 \mathrm{nM}$
Oligofectamine 2000

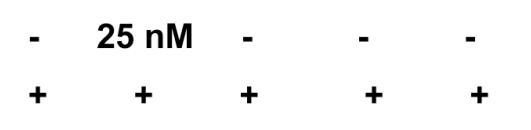


Figure 2

A

B

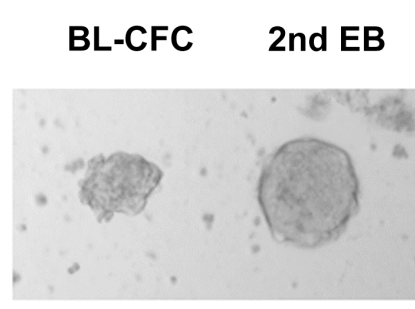

C

BL-CFC

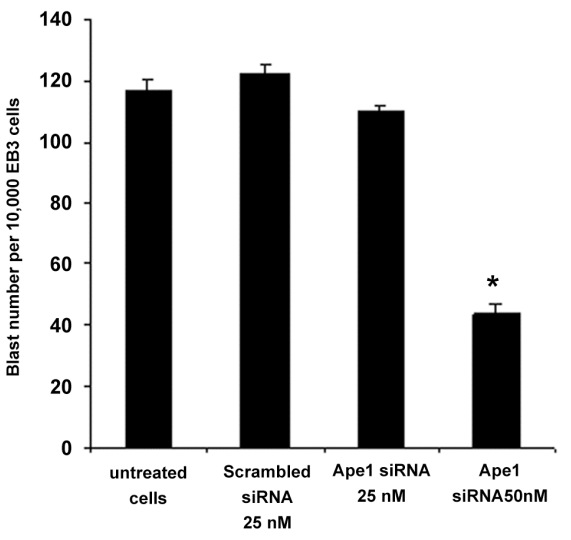

\section{BL-CFC 2nd EB}

Bry

Flk-1

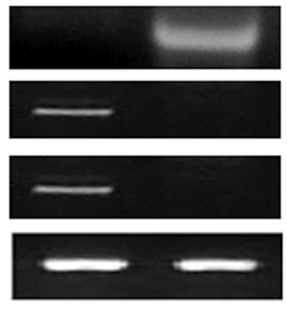

D

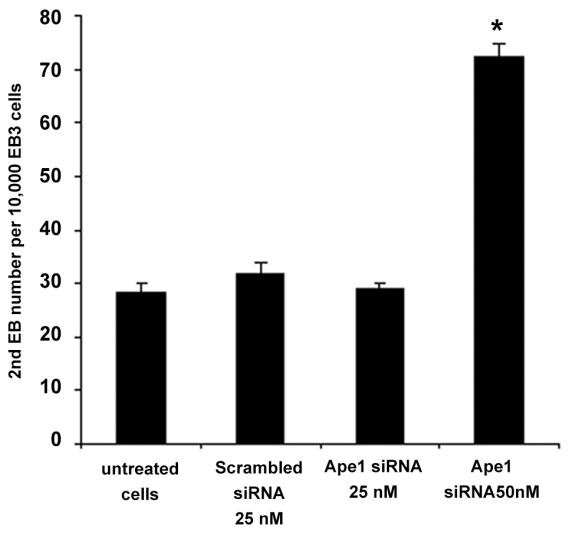


Figure 3

A

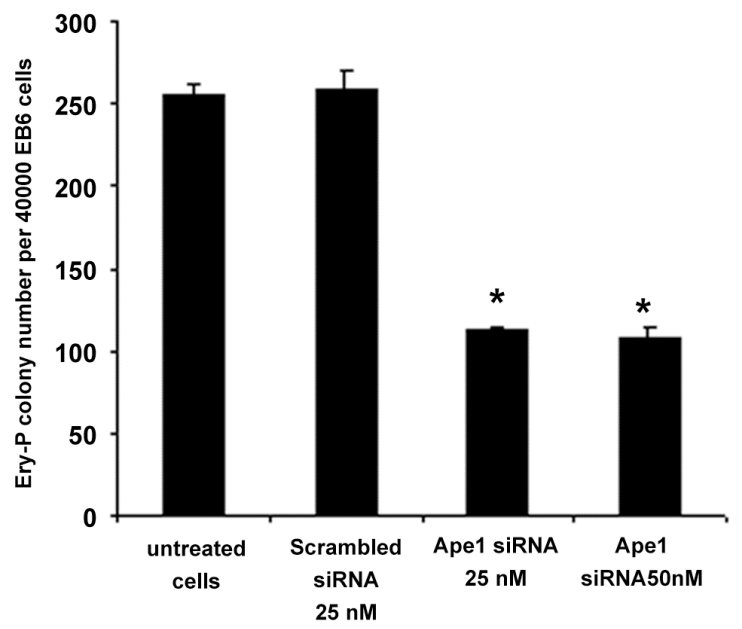

B

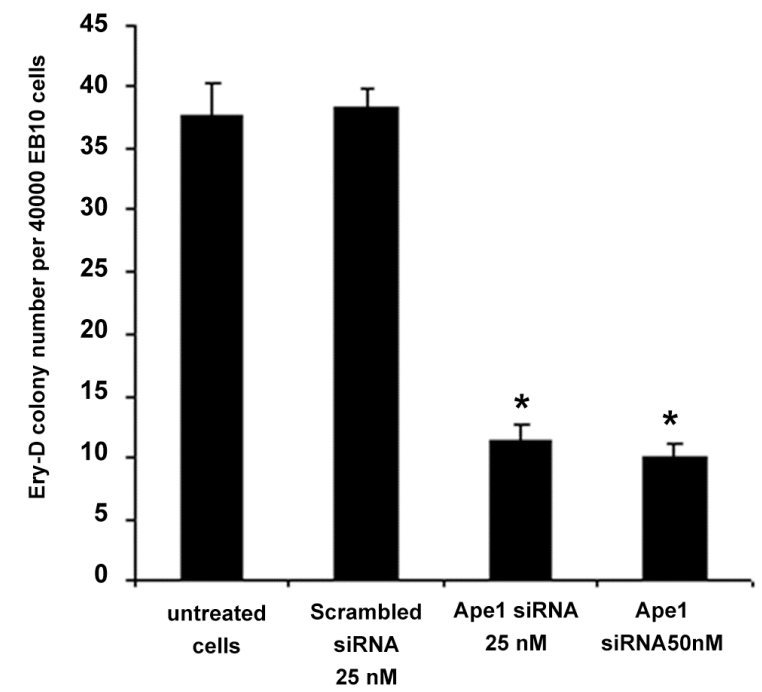

C

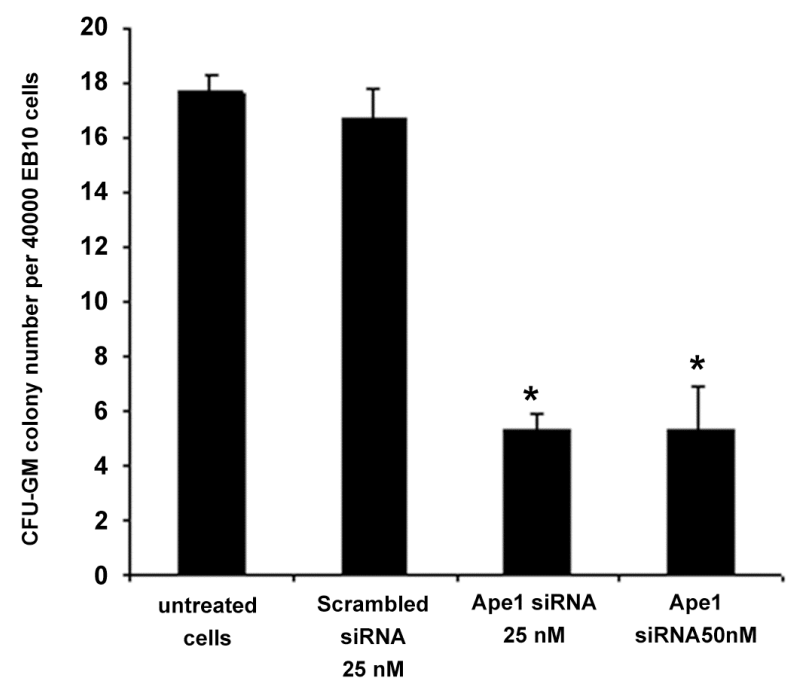


Figure 4
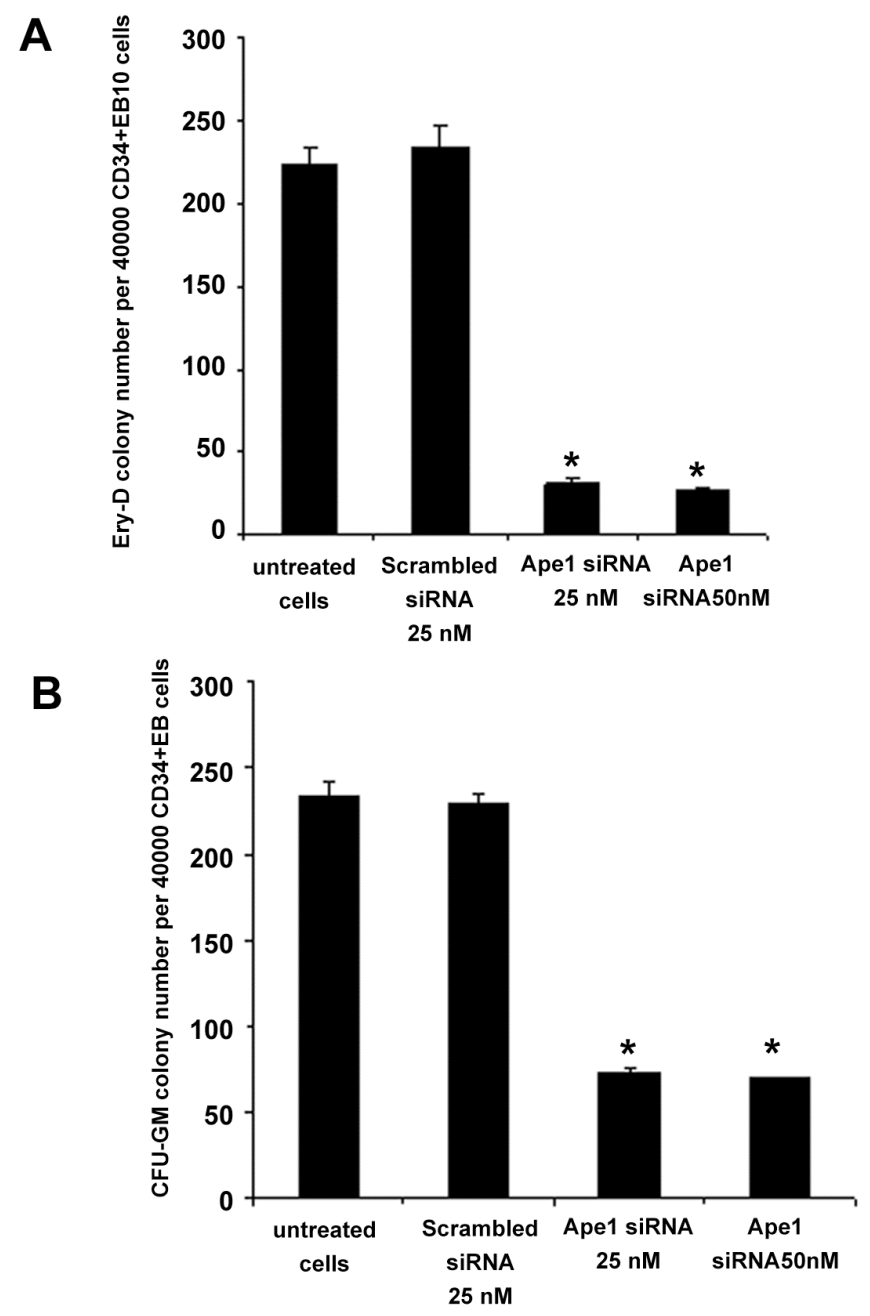

C

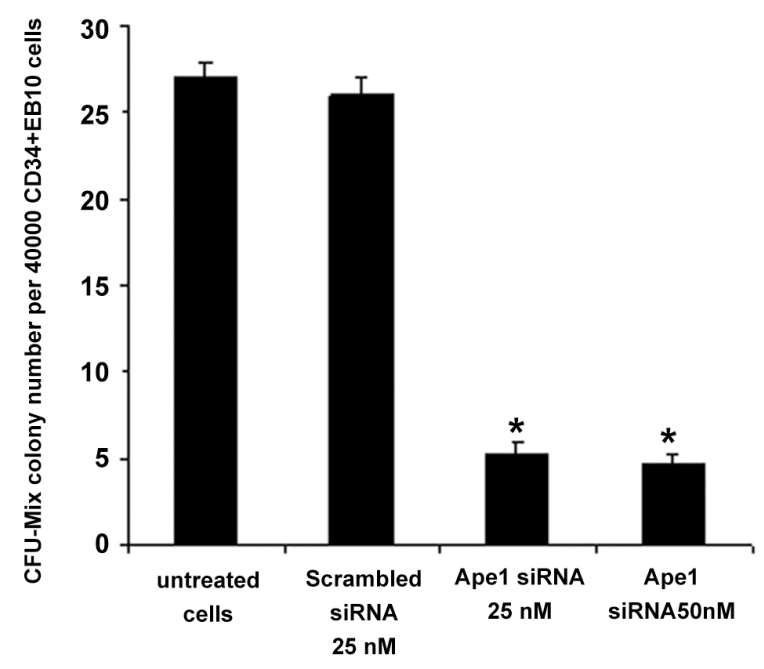


Figure 5

A

EB3 cells

A

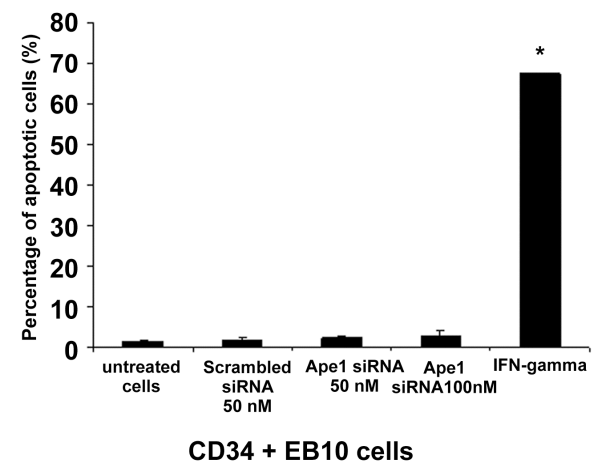

B

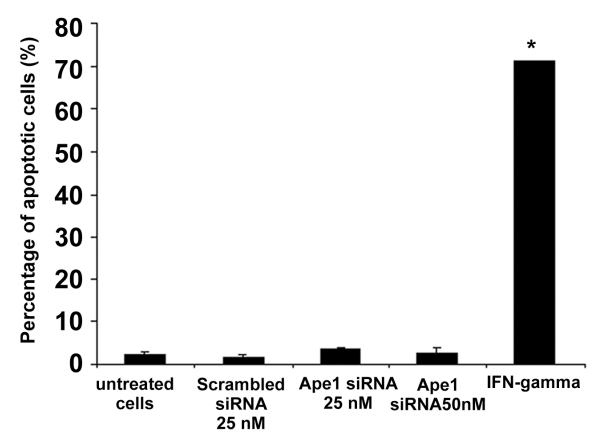

Figure 6

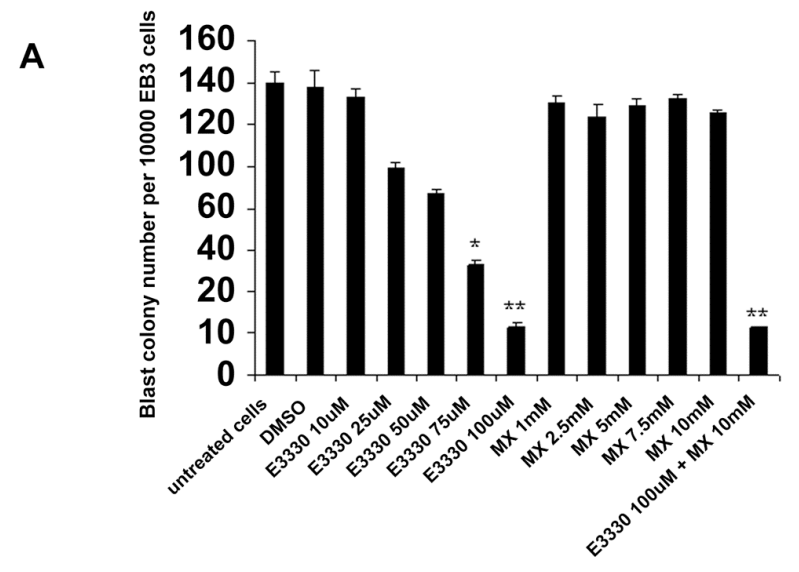

B

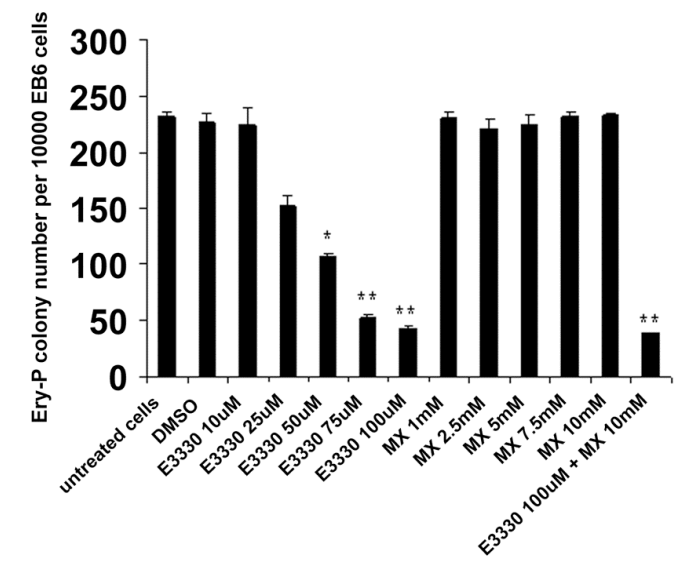


Figure 7

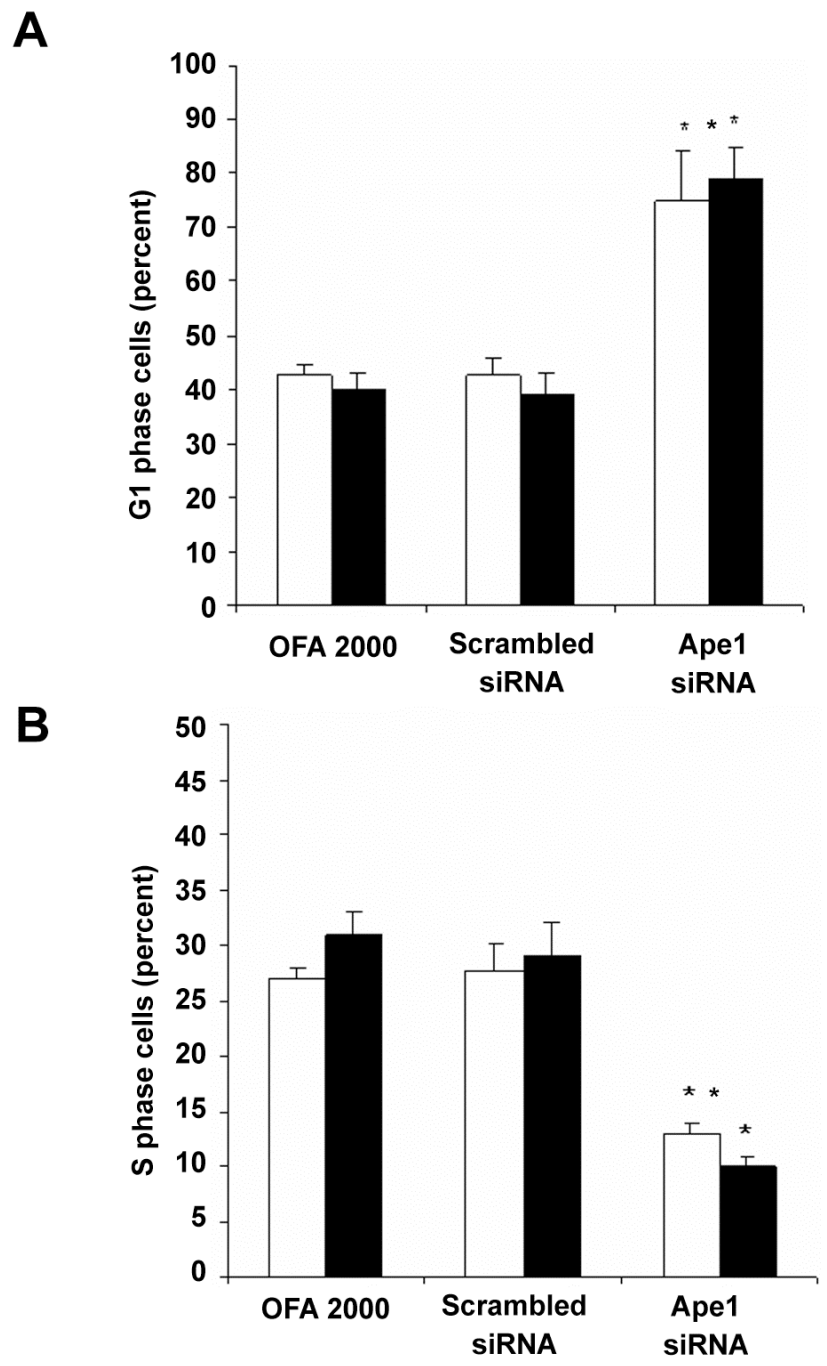

